\title{
Bimodal atomic force microscopy imaging of isolated antibodies in air and liquids
}

\author{
N. F. Martínez ${ }^{1}$, J.R. Lozano ${ }^{1}$, E.T. Herruzo ${ }^{1}$, F. Garcia ${ }^{1}$, \\ C. Richter ${ }^{2}$, T. Sulzbach ${ }^{2}$ and R.Garcia ${ }^{1, a}$ \\ ${ }^{1}$ Instituto de Microelectrónica de Madrid, CSIC, Isaac Newton 8, 28760 Tres Cantos, \\ Madrid, Spain \\ ${ }^{2}$ NanoWorld Services GmbH, Schottkystraße 10, 91058 Erlangen, Germany \\ ${ }^{a}$ Corresponding author, email: rgarcia@imm.cnm.csic.es
}

We develop a dynamic atomic force microscopy (AFM) method based on the simultaneous excitation of the first two flexural modes of the cantilever. The instrument, called bimodal AFM, allows us to resolve the structural components of antibodies in both monomer and pentameric forms. The instrument operates in both high and low quality factor environments, i.e., air an liquids. We show that under the same experimental conditions, bimodal AFM is more sensitive to compositional changes than amplitude modulation AFM. By using theoretical and numerical methods, we study the material contrast sensitivity as well as the forces applied on the sample during bimodal AFM operation. 


\section{Introduction.}

Atomic or molecular resolution images by atomic force microscopy (AFM) imply the application of forces of about $0.5 \mathrm{nN}$ on top of a few atoms. Individual covalent bonds within a crystal lattice readily sustain those forces by experiencing very small displacements in the sub-angstrom range. However, the individual noncovalent bonds that hold together the tertiary structure of proteins $(50-100 \mathrm{pN})$ may be broken by the AFM probe. This fact has prevented the observation of isolated biomolecules at molecular level.

Many attempts have been performed to render high resolution images of isolated proteins [1-8]. Cryogenic AFM has hinted the intramolecular structure of Y-shaped IgG antibodies [5]. There, the low temperatures enhanced the attachment of the molecules to the flat support and increased the molecular rigidity by suppressing the thermal motion. Ultra sharp single-walled carbon nanotube tips $(\sim 3 \mathrm{~nm})$ have also been applied to study antibodies and DNA [2]. Operating an amplitude modulation AFM (AM-AFM) in the attractive regime has also showed the Y-shape structure of antibodies in air [4, 6]. However, either the intrinsic limitations of cryo-AFM, the difficulties to fabricate ultra sharp nanotube probes [9] or the narrow instrumental window to access the attractive regime have severely limited the evolution and impact of the above approaches.

On the other hand, molecular resolution images have been achieved by imaging crystalline or semicrystalline two dimensional protein or lipid bilayer domains in liquid [10-13]. There, the close packing provided a mechanism to release the force exerted by the tip into vertical and lateral elastic deformations, so the molecular shape remains unchanged during imaging. Additionally, periodic structures enables the use of averaging procedures to improve resolution [14].

In conventional AM-AFM experiments (Figure 1(a)), the applied forces are usually reduced by using small free amplitudes (in order to maximize the amplitude range where cantilever oscillates in the attractive regime) and by working at relatively high average distances (set-point amplitude close to the free amplitude) [15]. Those conditions usually suppress compositional contrast in phase contrast images. Under the above conditions the tip-surface forces involve conservative or quasi-conservative processes which do not give rise to phase contrast in AM-AFM [16-18]. Thus, other AFM methods are needed to enhance compositional contrast while imaging soft biological samples at low forces.

Recently, several studies have proposed the use of either higher harmonics [1821] or modes [22-25] to enhance the sensitivity to tip-surface interactions. In particular, theoretical modelling by Rodriguez and Garcia has shown that, in the presence of mode coupling [26], the second mode of the cantilever is able to detect long range attractive force variations of $10 \mathrm{pN}$. Those simulations prompted the development of a new technique called bimodal AFM which consists on the simultaneous excitation of the two flexural modes of the cantilever, usually the first and the second (Figure 1(b)). This method opens two additional information channels (second mode amplitude and phase) with respect to conventional AM-AFM operation (monomodal excitation).

Experiments performed on conjugated molecular materials and proteins have showed a substantial compositional contrast with respect to AM-AFM and phase 
imaging [27-28]. Proksch has used the same method to image graphite sub-surface structures in air and desoxyrribonucleic acids in water [29]. Bimodal AFM imaging is also compatible with nanotomography techniques applied to polymers [30]. Stark et al. have used this method to minimize the cross-talk between mechanical and electrical interactions while imaging charge patterns in electrets [31]. Other recent applications include the imaging of absorbed protein monolayers [32]. A theoretical model shows [33] that both conservative and dissipative interactions are responsible for the material contrast observed in bimodal AFM imaging. The phase shift of the first mode is constrained by the feedback in the first mode amplitude while several parameters of the second mode are free to map compositional changes on the surface. However, many aspects of bimodal AFM operation must be addressed. Specifically in this contribution we analyze the potential of bimodal AFM for high resolution imaging of biomolecules in either liquids or air. we perform a comparison between tapping mode and bimodal AFM imaging of antibodies under the same applied forces. We also study which parameters of the microscope are more sensitive to detect material contrast in bimodal AFM.

\section{Experimental set-up.}

The experiments were carried out in both ambient and liquids with a hybrid AFM that includes commercial components (Nanoscope IV AFM controller and a multimode base from Veeco and a home-built bimodal excitation/detection unit (bimodal unit) [27]. The bimodal unit allow us to perform both the multifrequency excitation and the analysis of the cantilever oscillation signal. The unit provides four DC signals as outputs. These are the amplitudes and phase shifts of the first and second flexural modes, $A_{1}, A_{2}, \phi_{1}, \phi_{2}$. These signals can be introduced as external inputs to the AFM imaging software (Figure 1(c)). The photodiode signal of the amplitude of the first mode is fed back to the control unit to perform the distance control similarly as in AM-AFM. The flexural frequencies are determined by the standard method of recording the amplitude as a function of the excitation frequency and finding the peaks. The experiments in liquids were performed in a conventional fluid cell.

\section{Materials and methods.}

We have used both commercial and specially tailored cantilevers (NanoWorld, Germany) for bimodal AFM operation in air. The commercial cantilevers have force constant values of $\mathrm{k}=6-10 \mathrm{~N} / \mathrm{m}$ and first and second mode flexural frequencies $f_{l}=$ $110-120 \mathrm{KHz}$ and $f_{2}=650-700 \mathrm{KHz}$. The tailored cantilevers have been designed to enhance the response of the second eigenmode (Figure 2a). For imaging in liquids, we have used Olympus OMCL-RC800PSA cantilevers with nominal spring constant of $0.39 \mathrm{~N} / \mathrm{m}$. The cantilever spring constant for the commercial cantilevers was determined by using Sader's formula [34], while the bimodal cantilevers were calibrated by the thermal noise spectrum method $[35,36]$. The cantilever was mechanically excited by a piezo-actuator attached to the cantilever chip holder.

The photodiode sensitivity was first calibrated for contact mode and then recalibrated for first and second modes by considering the angle at the cantilever end with a continuous model [37]. This procedure is not valid for the tailored cantilevers because those cantilevers have not an uniform geometry as it is assumed in the model 
(rectangular cantilevers). We obtained calibration values of $95 \mathrm{~nm} / \mathrm{V}$ and $28 \mathrm{~nm} / \mathrm{V}$ for first and second modes.

Antibodies were deposited over a freshly cleaved piece of mica. They were prepared from a concentrated solution and diluted 1:100-1:1000 times until an homogenous deposition of antibodies over the mica was achieved. The antibodies were purchased from Sigma Aldrich (I8260 or A6029 for IgG or IgM respectively)

Antibodies are proteins that have well defined structures and binding sites. Those properties makes them good candidates to test the sensitivity and resolution of AFM methods [1-5]. We have imaged antibodies in monomer and pentameric forms. IgG is a Y-shaped protein that consists of four polypeptide chains arranged in three fragments, one Fc receptor and two identical Fab antigen-binding sites. The van der Waals length of each fragment is about $6 \mathrm{~nm}$. IgM has five Y-shaped monomers (inset of figure 4(b)), each of them having one Fc and two Fab fragments. Additionally, there is a small polypeptide chain (J-chain) that joins two consecutive Fc fragments.

\section{Theoretical model.}

Theoretical simulations have contributed to the understanding of cantilever-tip dynamics under the presence of tip-surface forces $[4,19,26,38,39,40]$. Here we simulate the bimodal AFM by solving the Euler-Bernouilli equation for a one dimensional beam that interacts with a surface and is externally excited at its first two eigenfrequencies $[26,41]$

$$
E I \frac{\partial}{\partial x^{4}}\left[w(x, t)+a_{1} \frac{\partial w(x, t)}{\partial t}\right]+\rho b h \frac{\partial^{2} w(x, t)}{\partial t^{2}}=-a_{0} \frac{\partial w(x, t)}{\partial t}+\delta(x-L) \mathbf{F}_{e x c}(t)+F_{t-s}(d)_{-}^{-}
$$

where $x$ is the spatial coordinate along the beam, $E$ the cantilever Young modulus, $I$ the area moment of inertia, $a_{1}$ the internal damping coefficient, $\rho$ the mass density, $b$ the width, $h$ the height and $L$ is the length of the rectangular cantilever; $a_{0}$ is the hydrodynamic damping. Finally, $w(x, t)$ is the time dependent transverse displacement of the cantilever. The excitation force under bimodal operation is $F_{\text {exc }}(t)=F_{1} \cos \omega_{1} t+$ $F_{2} \cos \omega_{2} t$, with $\omega_{1}$ and $\omega_{2}$ the normal bending frequencies and $F_{1}$ and $F_{2}$ the corresponding driving forces. The model considers long-range attractive conservative forces between tip and surface, modelled by the van der Waals expression [42]

$$
F_{t s}(d)=-\frac{H R}{6 d^{2}}
$$

where $H$ is the Hamaker constant related to the sample free surface energy and $R$ the effective tip radius. The instantaneous tip-sample distance is defined as $d(t)=z_{c}+$ $w(L, t)$ with $z_{c}$ the cantilever base position and $w(L, t)$ the tip displacement.

The solution of the Euler-Bernouilli equation can be Fourier-transformed in order to extract the amplitude and phase at first and second mode frequencies, according to 
$w(L, t)=A_{1} \cos \left(\omega_{1} t-\phi_{1}\right)+A_{2} \cos \left(\omega_{2} t-\phi_{2}\right)$

\section{Experimental results.}

\subsection{Tailored cantilevers for bimodal AFM operation in air}

We have designed several cantilevers to enhance the response of the second flexural mode (Figure 2(a)). The amplitude ratio of the different flexural modes can be modified by redistributing the mass along the cantilever length. Figure 2(b) shows a comparison of the frequency spectra of rectangular and tailored cantilevers. We compare the average values of the driving force (monomodal excitation) required to obtain a given value of the oscillation amplitude of the second flexural mode oscillation, here we set the target in of $1 \mathrm{~V}$ (table 1). For cantilevers of II, III and IV, the driving force required to reach the target output was $30-40 \mathrm{mV}$. For cantilevers I and V, a driving force $10 \mathrm{mV}$ was required to reach the target. In fact, cantilevers I and $\mathrm{V}$ were so sensitive that it was very hard to take images with them, because a change of $1 \mathrm{mV}$ in the driving force produced amplitude changes of $100 \mathrm{mV}$. This would make almost impossible to control the amplitude of the second mode in the required range of 0.2-1.4 $\mathrm{nm}$. On the other hand, the response of cantilevers II, III and IV did not show any appreciable differences with respect to those available commercially. This could be due to the fact that the quality factor of the second flexural mode is very high for both of them (800-1000).

\subsection{Comparison between amplitude modulation and bimodal AFM phase images}

To compare amplitude modulation and bimodal AFM phase images under the same conditions we have taken images of the same IgG antibodies deposited on mica and with the same cantilever. Figures 3(a) and 3(b) shows the tapping mode AFM images (AM-AFM) and Figures 3(d), 3(e), 3(f) the bimodal AFM images. In the comparison, we used identical values for the free amplitude of the first mode $A_{01}$ and for the same set-point amplitude, those values were respectively $A_{01}=22 \mathrm{~nm}$ and $A_{s p}=21.2$. For bimodal AFM operation we added a second mode free amplitude of $A_{02}=0.7 \mathrm{~nm}$. Figure 3(c) shows AM-AFM and bimodal AFM amplitude oscillations.

The topography images given by both AM-AFM and bimodal AFM reveal featureless objects on the mica surface (Figures 3(a) and 3(d)). Furthermore, the phase image corresponding to the first mode does not reveal any kind of contrast between the protein and the mica surface (Figures 3(b) and 3(e)). The lack of contrast in the above phase images is due to the imaging conditions that were chosen to minimize tipantibody forces. This was achieved by using set-point values very close to the free amplitude. Consequently, the imaging was dominated by conservative tip-molecule forces. As it was stated previously, conservative forces do not give rise to material contrast in the phase images of the $1^{\text {st }}$ mode.

On the other hand, the phase image of the second mode does reveal three structures that could be easily linked to the three fragments of the IgG antibody (Fig. 3(f)). The phase image resolves the three lobes of the structure with a separation between peak lobes of 7.1 and $7.8 \mathrm{~nm}$. 


\subsection{Bimodal AFM imaging of antibodies in air}

Figure 4 shows topography and phase images ( $2^{\text {nd }}$ mode) of $\operatorname{IgG}$ antibodies acquired with a Bimodal AFM. Two of the objects resemble the Y-shape of IgG antibodies (termed as antibody 1 and antibody 2). The topography (Figure 4(a)) of antibody 1 shows the characteristic three lobes of $\operatorname{IgG}$ (see inset for an scheme of $\operatorname{IgG}$ ), while antibody 2 just shows a triangular shape. On the contrary, the bimodal AFM phase image (Figure 4(b)) resolves the three fragments of the molecule for both antibodies. In order to make a detailed comparison, topographic and bimodal AFM phase cross-sections are plotted for each antibody (Figures 5(a), 5(b), 5(c) and 5(d)). The comparison shows that for both antibodies the bimodal AFM phase image gives better lateral resolution than the corresponding topographic image.

The four Fab fragments (two per antibody) give very similar phase values $\left(3.8^{\circ}\right.$ $\left.4.2^{\circ}\right)$. However, we obtain a noticeable difference between the phase shift values of the Fc fragments $\left(\sim 1.2^{\circ}\right)$. The difference could be attributed to small differences between the morphologies of the Fc fragments upon deposition. This interpretation is consistent with the differences observed in the apparent lengths of antibodies 1 and 2. So that, differences in topographic lateral dimensions could be explained.

Bimodal AFM phase images reveal the monomer components of individual IgM antibodies deposited on mica (Figure 6). We observe that the lateral size of the antibodies as measured the AFM image is slightly larger than the nominal values (35 $\mathrm{nm}$ vs. $25 \mathrm{~nm}$ ). This broadening could be a convolution effect originated by the tip's finite size.

The above images have been obtained with a cantilever spring constant of 8.7 $\mathrm{N} / \mathrm{m}$, free amplitude values for first and second modes of $A_{01}=21.4 \mathrm{~nm}$ and $A_{02}=0.4$ $\mathrm{nm}$ respectively. The set-point amplitude was $A_{s p}=20.1 \mathrm{~nm}$.

\subsection{Bimodal AFM imaging of antibodies in liquids}

In conventional AFM fluid cells the mechanical excitation drives the cantilever, the fluid and the fluid cell. The resulting frequency spectrum shows many peaks that bury the genuine cantilever resonances. This forest of peaks phenomenon [43] is shown in the inset of Figure 7(a). It is difficult to conclude which of these peaks is closer to the true resonances of the cantilever. To determine the cantilever's eigenmodes in fluids we measured the thermal noise spectrum. In this way, the first and second flexural modes (arrows in the inset of Figure 7(a)) are found at $22.49 \mathrm{kHz}$ and $146.98 \mathrm{kHz}$ respectively.

For imaging IgM antibodies we have used a first mode free amplitude of $A_{01}=$ $9.1 \mathrm{~nm}$ and a second mode free amplitude of $A_{02}=1.9 \mathrm{~nm}$. The set-point amplitude was $6.0 \mathrm{~nm}$. Figure 7(a) shows the bimodal AFM phase image of IgM antibodies in water. We can see some individual antibodies (marked by circles) as well as multiple aggregates and smaller objects that could be single separate monomers. Figures 7(b), 7(c) and 7(d) show a comparison among topography, first mode phase and second mode phase images of another individual IgM. Topography and first mode phase images do not resolve the inner structure of the protein, however, the phase image of the second mode do distinguish the different monomers and the overall pentameric shape of 
the antibody. The apparent lateral size of the pentamers as given by the AFM image is $37 \mathrm{~nm}$.

\section{Simulations of bimodal AFM dynamics}

The numerical simulations have been performed for a cantilever with length $L$, width $b$ and thickness $h$ of $225 \mu \mathrm{m}, 40 \mu \mathrm{m}, 1.8 \mu \mathrm{m}$, respectively. The Young modulus and mass density were respectively $170 \mathrm{GPa}$ and $2320 \mathrm{~kg} / \mathrm{m}^{3}$. The force constants, resonance frequencies and quality factors of the first two flexural modes were respectively $0.9 \mathrm{~N} / \mathrm{m}, 35.2 \mathrm{~N} / \mathrm{m}, 48.913 \mathrm{kHz}, 306.194 \mathrm{kHz}, 255$ and 1000. The tip radius $R$ was $20 \mathrm{~nm}$. The simulations have been calculated for two different materials, $\mathrm{SiO}_{2}$-air-water-mica $\left(H=4.7 \times 10^{-20} \mathrm{~J}\right)$ and $\mathrm{SiO}_{2}$-air-mica $\left(H=9.03 \times 10^{-20} \mathrm{~J}\right)$ respectively.

Figure 8 shows the dependence of $A_{1}, A_{2}, \phi_{1}, \phi_{2}$ on the average tip-surface distance. The behaviour of the amplitude and phase shift of the first mode does not seem to depend on the sample's Hamaker values (Figures 8(a) and 8(c)). In particular, $A_{1}$ varies rather linearly with the tip-surface distance. This property makes the amplitude of the first mode a suitable feedback parameter to track topography in bimodal AFM. In addition, the curves do not show any noticeable dependence on the sample's Hamaker constant. On the other hand, the amplitude and phase shift curves of the second flexural mode (Figures 8(b) and 8(d)) show a marked difference with the Hamaker values. This property supports the use of the second mode parameters in bimodal AFM to extract information on material properties.

\subsection{Material contrast sensitivity in bimodal AFM operation}

To analyze the sensitivity of bimodal AFM operation to detect compositional changes we represent the differences in the microscope parameters $\left(A_{2}, \phi_{1}, \phi_{2}\right)$ (see Fig. 8 ) against the set-point amplitude $A_{l}$ (Figure 9). The material contrast is defined as the difference observed in any of the microscope parameters for two Hamaker constant values at a given set-point amplitude. The dashed lines are the experimental noise measured in our experimental set-up. As it was predicted [26], $\phi_{1}$ shows no material contrast above the noise level because of the absence of dissipation in the tip-surface forces (Figure 9(a)). The small contrast is attributed to numerical errors in the simulations. On the other hand, the parameters of the second mode do show material contrast well above the noise level [26, 33]. Figures 9(b) and 9(c) show a maximum in the contrast at intermediate set-point amplitudes. Consequently, those set-point values define the optimal conditions for material contrast imaging. The value of that Signal to Noise Ratio (SNR) is about 200 for $\phi_{2}$ and 3.6 for $A_{2}$.

The origin of the material contrast observed in bimodal AFM operation is due to the ability to detect conservative and nonconservative interactions. In contrast, conservative forces in AM-AFM do not give material contrast in the phase shift signal because the restrictions imposed by the feedback mechanism [33]. 
As in regular tapping-mode AFM operation, the non-linear nature of the interaction makes hard the determination of the sample's Young modulus or Hamaker constant from the experimental parameters. That is the reason why numerical calculations are widely used to simulate the tip-surface dynamics in theoretical AFM analyses [44].

To compare the material contrast obtained in amplitude and phase shifts $A_{2}$ and $\phi_{2}$, we have normalized the maximum contrast obtained in Figures 9(b) and 9(c) to their respective ranges of variation $\left(A_{02}=0.6 \mathrm{~nm}\right.$ for $A_{2}$ and $90^{\circ}$ for $\left.\phi_{2}\right)$ resulting $\Delta A_{2, \max } / 0.6$ $\mathrm{nm}=0.14$ and $\Delta \phi_{2, \max } / 90^{\circ}=0.11$. The comparison reveals a similar sensitivity for both parameters. However, the changes observed in the amplitude $0.08 \mathrm{~nm}$ are very close to the noise level $(\sim 0.04 \mathrm{~nm})$. The above does not occur for the phase shift variations.

Figures 9(d) and 9(e) show $A_{2}$ and $\phi_{2}$ images of a silicon sample covered by sexythiophene molecules. We can distinguish the same features in both images, thus revealing that compositional sensitivity is similar in both channels. In order to check the contrast of the two images, a profile (dashed line in Figures 9(d) and 9(e)) is depicted. In Figure 9(f) the $A_{2}$ image gives a positive contrast of $0.2 \mathrm{~nm}$, while $\phi_{2}$ image gives a negative contrast of $9.1^{\circ}$. Experimental conditions were: $k=8.5 \mathrm{~N} / \mathrm{m}, A_{01}=18.1 \mathrm{~nm}$, $A_{02}=1.8 \mathrm{~nm}$ with $A_{s p} / A_{01}=95 \%$.

\subsection{Estimation of the force applied on the sample surface}

Unlike in static AFM, direct measurements of the forces applied on the sample surface are not possible in dynamic AFM. However, there are several methods to reconstruct the value of the interaction from dynamic force curves, i.e., amplitude and phase shift versus distance curves in AM-AFM and frequency shift versus distance in FM-AFM [45-52]. The rationale for most of the above methods relies on inverting the integral equation deduced from $2^{\text {nd }}$ Newton law by averaging over one period of oscillation. In the integral equations the interaction force appears multiplied by the instantaneous tip deflection or its higher derivatives. This makes hard to extract the force as a function of the experimental parameters. We remark that the above methods have assumed a point-mass model for the cantilever, as a consequence all the higher modes of the cantilever but the fundamental have been neglected. This seems to be a reasonable approximation in bimodal AFM because $A_{02} / A_{01}<<1$.

Here, we apply the method developed by Hölscher in Ref. [49]. This method extracts the force at the minimum tip-surface distance from both amplitude and phase shift curves. In this case we choose $A_{l}$ and $\phi_{l}$ as the tapping amplitude and phase values, respectively, since we neglect the second mode tip-surface dynamics. According to Hölscher's formula, the maximum force calculated at resonance as the force at the minimum distance, $d_{\min }$, is

$$
F_{t s}\left(d_{\min }\right)=-\frac{F_{1}}{\sqrt{2}} \frac{\partial}{\partial d_{\text {min }}} \int_{l_{\min }}^{l_{\min }+2 A_{1}\left(d_{\min }\right)} d z \sqrt{\frac{A_{1}(z)}{z-d_{\text {min }}}} \cos \phi_{1}(z)
$$


where $F_{1}=k_{1} A_{01} / Q_{1}$ is the first mode driving force $\left(k_{1}\right.$ and $Q_{1}$ are the spring constant and quality factor for the first mode, respectively). The values for $z$ in the numerical integration of Eq. 4 have to be taken from the corresponding amplitude and phase shift dynamic force curves, at the minimum distance, $d_{\min }=z_{c}-A_{l}$.

Figure 10(a) shows the minimum tip-surface distance as calculated by numerical simulations for both bimodal and monomodal (conventional) excitation methods. The tip-surface distance hardly changes by the introduction of the bimodal excitation. Consequently, the maximum force per oscillation will hardly change by introducing the second mode excitation. This in turns supports the use of algorithms based on pointmass models to reconstruct the force in bimodal AFM operation. The reconstructed force lies below $120 \mathrm{pN}$ (Fig. 10(b)). The force varies from 0 (no interaction) to a maximum of $120 \mathrm{pN}$. Most of the experimental data shown here were acquired at an amplitude ratio $A_{1} / A_{01}=0.95$ (black cross) which gives a maximum forces below 100 $\mathrm{pN}$.

\section{Summary}

We have presented a dynamic force microscopy method based on the simultaneous excitation of the first two flexural modes of the cantilever. The performance and the potential of the instrument for biology applications has been characterized by imaging isolated antibodies in air and water. The instrument resolves the Fc and Fab fragments in single antibodies as well as the individual monomers in pentameric antibodies.

We have also compared the compositional sensitivity and spatial resolution of amplitude modulation and bimodal AFM methods. Under experimental conditions aimed to minimize tip-surface forces, bimodal AFM phase images show higher compositional contrast and spatial resolution than amplitude modulation AFM topography and phase images.

One of the advantages of bimodal AFM is that makes compatible high resolution imaging of isolated biomolecules at very low forces. Routine bimodal AFM imaging can be performed by applying maximum forces below $100 \mathrm{pN}$. The force exerted by the tip on the biomolecule has been estimated by using theoretical methods.

We have also characterized the sensitivity of the different bimodal AFM parameters to detect material contrast. The phase shift of the second flexural mode is the parameter that gives the highest material contrast.

Acknowledgements. This work was financially supported by the European Commission (FORCETOOL, NMP4-CT-2004-013684), Ministerio de Educación y Ciencia (MAT2006-03833) and Comunidad de Madrid (S-0505/MAT/0283). 
Table 1. Resonance frequencies and driving forces to obtain a second mode oscillation amplitude of $1 \mathrm{~V}$.

\begin{tabular}{lcc}
\hline \multicolumn{1}{c}{ Cant. Type } & $\left\langle\mathbf{f}_{2}(\mathbf{k H z})\right\rangle$ & $\langle$ driving force $(\mathbf{m V})\rangle$ \\
\hline I & 491.6 & 8 \\
\hline II & 375.2 & 32 \\
\hline III & 484.1 & 32 \\
\hline IV & 388.2 & 42 \\
\hline V & 413.1 & 9 \\
\hline Commercial & 703.5 & 48
\end{tabular}




\section{References}

[1] Fritz M, Radmacher M, Cleveland J P, Allersma M W, Stewart R J, Gieselmann R, Janmey P, Schmidt C F and Hansma P K 1995 Langmuir 11 3529-35

[2] Hafner J H, Cheung C L and Lieber C M 1999 Nature 398 761-2

[3] Hinterdorfer P and Dufrene Y F 2006 Nat. Meth. 3 347-55

[4] San Paulo A and Garcia R 2000 Biophys. J. 78 1599-605

[5] Shao Z F and Zhang Y Y 1996 Ultramicroscopy 66 141-52

[6] Thomson N H 2005 Ultramicroscopy 105 103-10

[7] Horber J K H and Miles M J 2003 Science 302 1002-5

[8] Muller D J and Engel A 2007 Nat. Protocol. 2 2191-7

[9] Martinez J, Yuzvinsky T D, Fennimore A M, Zettl A, Garcia R and Bustamante C 2005 Nanotechnology 16 2493-6

[10] Ando T, Kodera N, Takai E, Maruyama D, Saito K and Toda A 2001 Proc. Natl. Acad. Sci 98 12468-72

[11] Higgins M J, Polcik M, Fukuma T, Sader J E, Nakayama Y and Jarvis S P 2006 Biophys. J. 91 2532-42

[12] Hoogenboom B W, Hug H J, Pellmont Y, Martin S, Frederix P L T M, Fotiadis D and Engel A 2006 Appl. Phys. Lett. 88 193109-3

[13] Scheuring S, Seguin J, Marco S, Levy D, Robert B and Rigaud J-L 2003 Proc. Natl. Acad. Sci 100 1690-3

[14] Engel A and Muller D J 2000 Nat Struct Mol Biol 7 715-8

[15] García R and San Paulo A 1999 Phys. Rev. B 604961

[16] Garcia R, Gomez C J, Martinez N F, Patil S, Dietz C and Magerle R 2006 Phys. Rev. Lett. 97 016103-4

[17] Martinez N F and Garcia R 2006 Nanotechnology 17 S167-S72

[18] Legleiter J and Kowalewski T 2005 Appl. Phys. Lett. 87 163120-3

[19] Preiner J, Tang J, Pastushenko V and Hinterdorfer P 2007 Phys. Rev. Lett. 99 046102-4

[20] Sahin O, Quate C F, Solgaard O and Atalar A 2004 Phys. Rev. B 69

[21] Stark M, Stark R W, Heckl W M and Guckenberger R 2000 Appl. Phys. Lett. 77 3293-5

[22] Crittenden S, Raman A and Reifenberger R 2005 Phys. Rev. B 72 235422-13

[23] Dareing D W, Thundat T, Jeon S and Nicholson M 2005 J. Appl. Phys. 97 084902-6

[24] Minne S C, Manalis S R, Atalar A and Quate C F 1996 Appl. Phys. Lett. 68 1427-9

[25] Sugimoto Y, Innami S, Abe M, Custance O and Morita S 2007 Appl. Phys. Lett. 91 093120-3

[26] Rodriguez T R and Garcia R 2004 Appl. Phys. Lett. 84 449-51

[27] Martinez N F, Patil S, Lozano J R and Garcia R 2006 Appl. Phys. Lett. 89 153115-3

[28] Patil S, Martinez N F, Lozano J R and Garcia R 2007 J. Molec. Recognit. 20 516-23

[29] Proksch R 2006 Appl. Phys. Lett. 89 113121-3

[30] Dietz C, Zerson M, Riesch C, Gigler A M, Stark R W, Rehse N and Magerle R 2008 Appl. Phys. Lett. 92 143107-3 
[31] Stark R W, Naujoks N and Stemmer A 2007 Nanotechnology 18065502

[32] Platz D, Tholen E A, Pesen D and Haviland D B 2008 Appl. Phys. Lett. 92 153106-3

[33] Lozano J R and Garcia R 2008 Phys. Rev. Lett. 100 076102-4

[34] Sader J E, Chon J W M and Mulvaney P 1999 Rev. Sci. Instrum. 70 3967-9

[35] Butt H J and Jaschke M 1995 Nanotechnology 6 1-7

[36] Levy R and Maaloum M 2002 Nanotechnology 13 33-7

[37] Schaffer T E 2005 Nanotechnology 16 664-70

[38] Melcher J, Hu S and Raman A 2007 Appl. Phys. Lett. 91 053101-3

[39] Stark R W, Schitter G, Stark M, Guckenberger R and Stemmer A 2004 Phys. Rev. B 69085412

[40] Solares S D 2007 J. Phys. Chem. B $1112125-9$

[41] Stark R W and Heckl W M 2000 Surf. Sci. 457 219-28

[42] Israelachvili J 2005 Intermol. and Surf. Forces (London: Elsevier Academic Press)

[43] Schaffer T E, Cleveland J P, Ohnesorge F, Walters D A and Hansma P K 1996 J. Appl. Phys. 80 3622-7

[44] Raman A, Melcher J and Tung R 2008 Nano Today 3 20-7

[45] Giessibl F J 1997 Phys. Rev. B 5616010

[46] Durig U 1999 Appl. Phys. Lett. 75 433-5

[47] Stark M, Stark R W, Heckl W M and Guckenberger R 2002 Proc. Natl. Acad. Sci 99 8473-8

[48] Sader J E and Jarvis S P 2004 Appl. Phys. Lett. 84 1801-3

[49] Holscher H 2006 Appl. Phys. Lett. 89 123109-3

[50] Holscher H and Schwarz U D 2007 Intern. J. Non-Linear Mechs. 42 608-25

[51] Hu S and Raman A 2007 Appl. Phys. Lett. 91 123106-3

[52] Lee M H and Jhe W H 2006 Phys. Rev. Lett. 97 036104-4 


\section{Figure Captions}

Figure 1. Comparison between amplitude modulation and bimodal AFM. (a) AM-AFM (monomodal excitation). (b) Bimodal AFM. (c) Schematics of the bimodal AFM instrument. The bimodal excitation/detection unit performs the multifrequency excitation and the multicomponent signal processing while the control unit runs the feedback.

Figure 2. (a) Cantilevers designed for enhancing the second flexural mode response. (b) Comparison of the frequency response between commercial and tailored cantilevers.

Figure 3. Comparison between AM-AFM and bimodal AFM images of IgG antibodies. (a) Topography and (b) phase images of an IgG obtained in AM-AFM. (c) Tip oscillation in AM-AFM (top) and bimodal AFM (bottom). (d) Topography in bimodal AFM. (e) Phase shift image of the first mode in bimodal AFM. (f) Bimodal AFM phase image $\left(2^{\text {nd }}\right.$ mode) of the same antibody. The image shows a Y shaped object.

Figure 4. Bimodal imaging of IgG antibodies. (a) Topography. (b) Second mode phase image (b). Two objects are identified as IgG proteins (termed as 1 and 2). The inset shows a scheme of the IgG antibody structure.

Figure 5. Cross-sections along the arrows shown in Figure 4(a) and 4(b) for antibodies 1 and 2. (a) is the topography and (b) the phase shift $\left(2^{\text {nd }}\right.$ mode) cross-sections corresponding to antibody 1 . (c) is the topography and (d) the phase shift ( $2^{\text {nd }}$ mode) cross-sections corresponding to antibody 2 . The darker cross-sections correspond to the darker line in Fig. 4.

Figure 6. (a) Topography image of a region with a homogenous deposition of the antibodies. (b) Topography image of an isolated antibody. The antibody shows an overall pentagonal shape but there is no hint on the monomer positions. (c) Scheme of the IgM antibody. (d), (e) and (f) Bimodal AFM phase images $\left(2^{\text {nd }}\right.$ mode) of three different antibodies. The above images reveal the monomer components, they also hint the position of the J-chain.

Figure 7. (a) Bimodal AFM phase images ( $2^{\text {nd }}$ mode) of $\operatorname{IgM}$ antibodies in water. The objects that show a pentagonal shape are marked by circles. The inset shows the frequency spectrum of a commercial cantilever in water. The dashed lines indicate the frequencies of first and second flexural modes of the cantilever. They were determined by measuring the thermal noise spectrum. (b) Topography of an isolated antibody. (c) First mode phase image and (d) Bimodal AFM phase image $\left(2^{\text {nd }}\right.$ mode) of the same antibody.

Figure 8. Amplitude and phase shift curves for two different materials (Hamaker values of $4.7 \times 10^{-20} \mathrm{~J}$, open dots; $9.03 \times 10^{-20} \mathrm{~J}$, dark dots). (a) Amplitude curve of the first mode. (b) Amplitude curve of the second mode. (c) Phase shift curve of the first flexural mode.

(d) Phase shift curve of the second flexural mode. 
Figure 9. Material contrast sensitivity in bimodal AFM. (a) Dependence of the $1^{\text {st }}$ mode phase shift contrast on the set-point amplitude. (b) Dependence of the bimodal AFM phase shift ( $2^{\text {nd }}$ mode) contrast on the set-point amplitude. (c) Dependence of the bimodal AFM amplitude ( $2^{\text {nd }}$ mode) contrast on the set-point amplitude. The materials have been simulated by using two Hamaker values $\left(H=4.7 \times 10^{-20} \mathrm{~J}\right.$ and $\left.9.03 \times 10^{-20} \mathrm{~J}\right)$. The dashed lines represent the experimental noise. Experimental $A_{2}(\mathrm{~d})$ and $\phi_{2}$ (e) images of sexythiophene molecules on silicon. (f) Cross section along the dashed lines of Fig. 9d.

Figure 10. (a) Minimum tip-surface distance for bimodal (dark dots) and monomodal (open dots) excitations as a function of the amplitude ratio. (b) Reconstructed tipsurface forces as a function of the amplitude ratio. The simulations were performed with $H=9.03 \times 10^{-20} \mathrm{~J}$ and $R=20 \mathrm{~nm}$. 
Figure 1
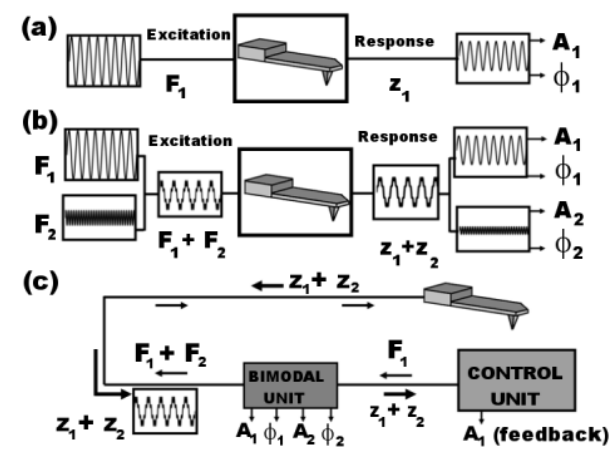

Figure 2
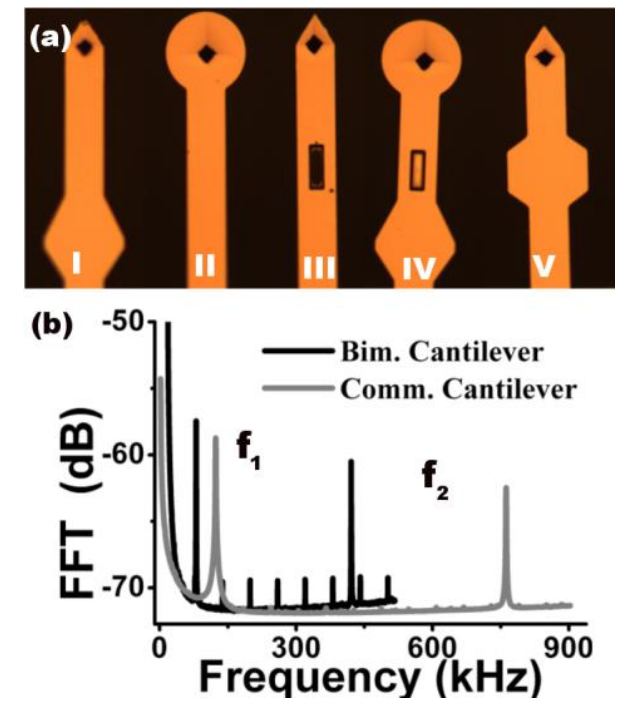


\section{Figure 3}

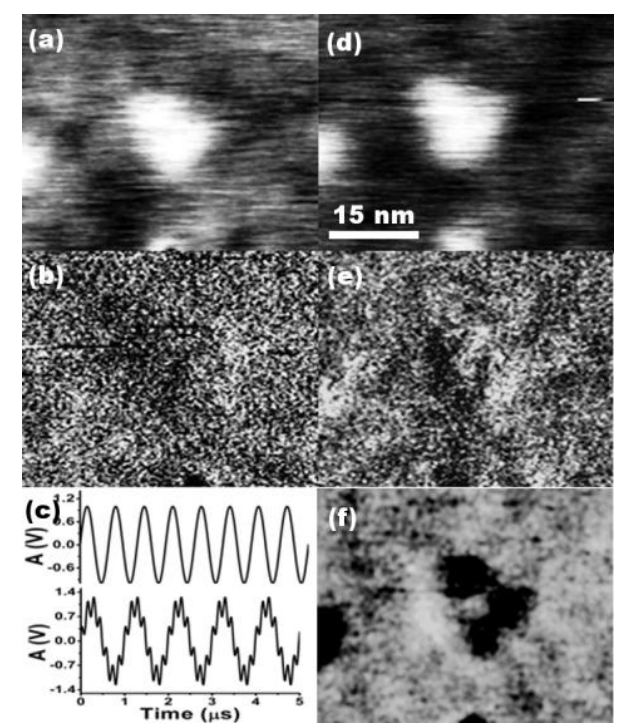

Figure 4

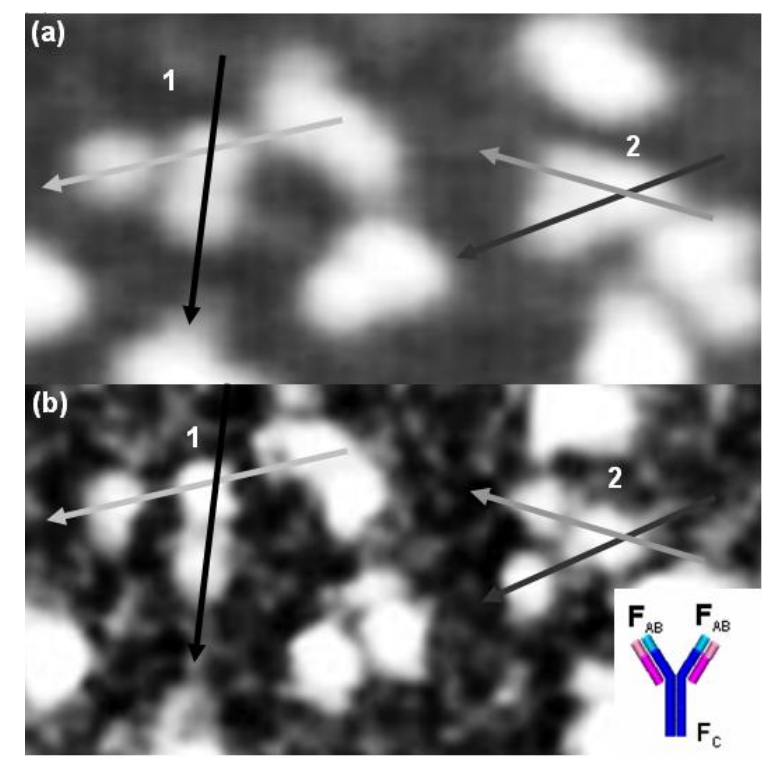


Figure 5
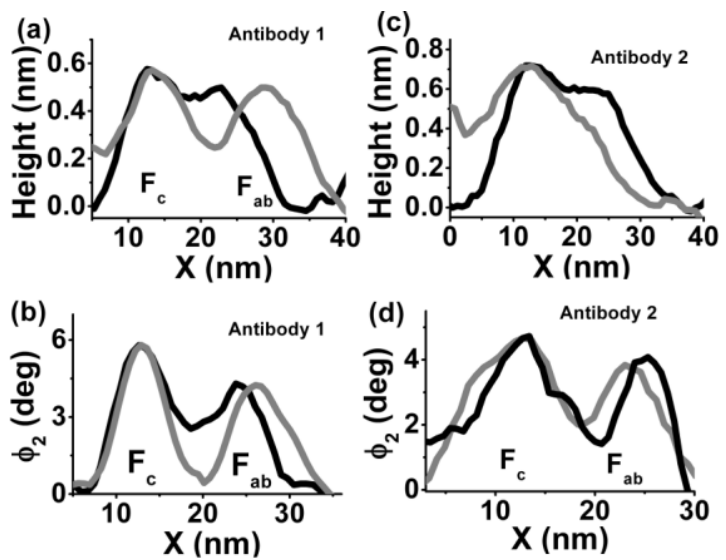

Figure 6

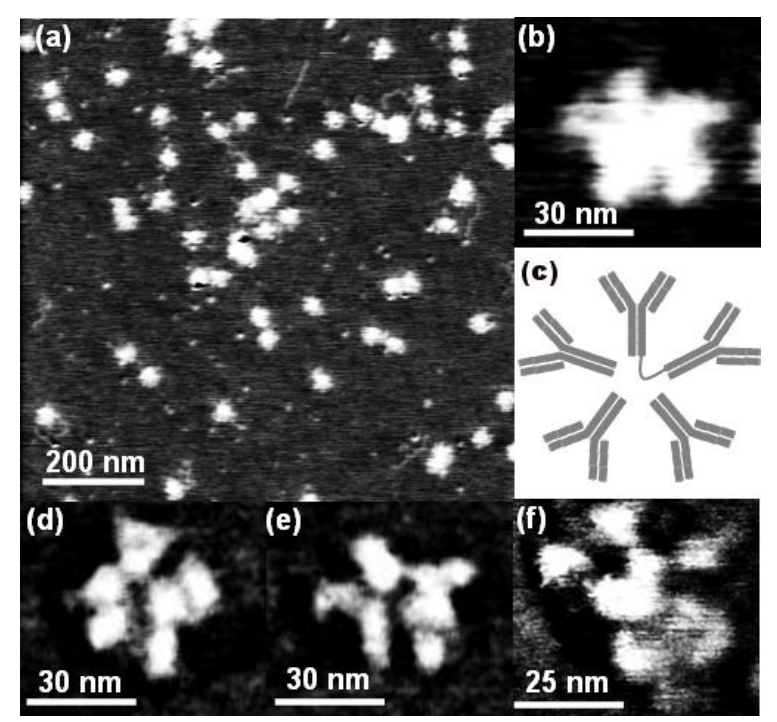

Figure 7

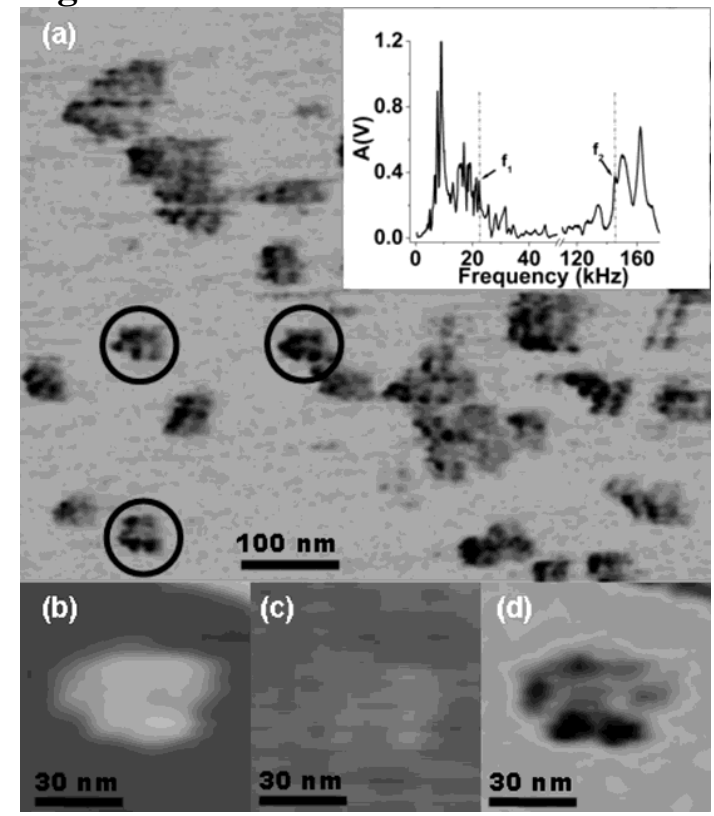


Figure 8
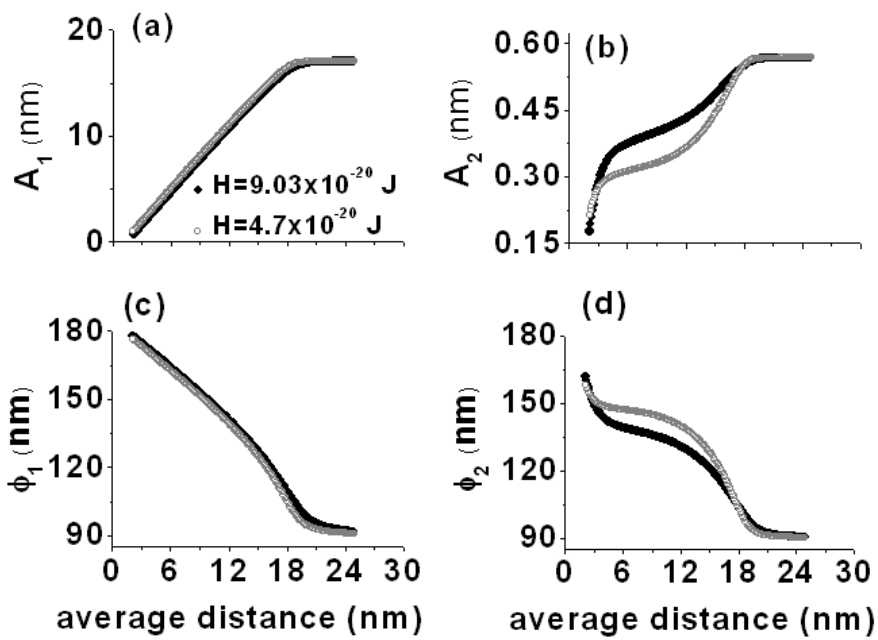

Figure 9
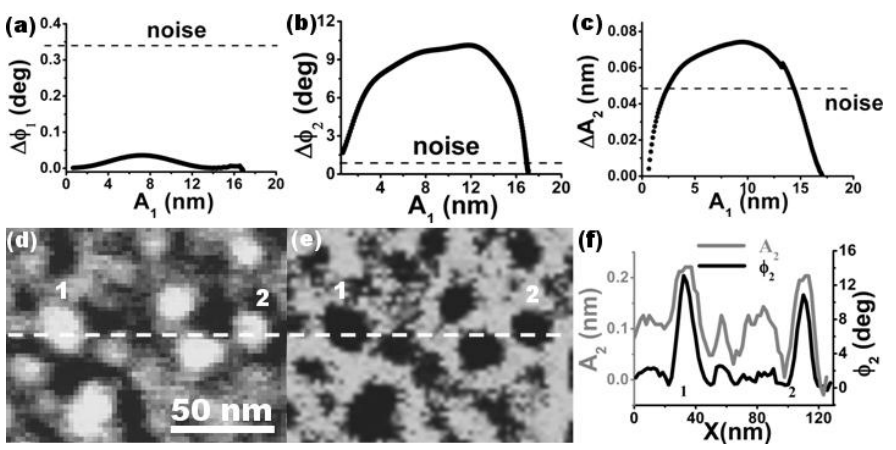

Figure 10

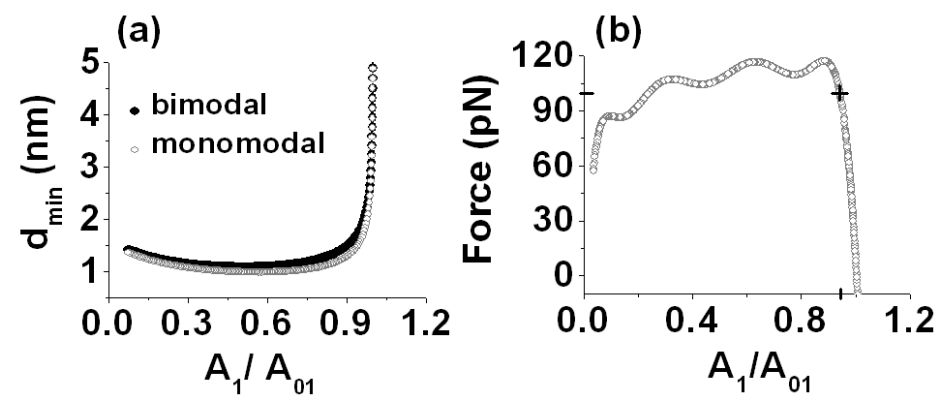

